\title{
Proactive planning and valuation of transmission investments in restructured electricity markets
}

\author{
Enzo E. Sauma • Shmuel S. Oren
}

Published online: 8 December 2006

(C) Springer Science+Business Media, LLC 2006

\section{Erratum to: J Regul Econ DOI: 10.1007/s11149-006-9003-y}

\begin{abstract}
Traditional methods of evaluating transmission expansions focus on the social impact of the investments based on the current generation stock which may include firm generation expansion plans. In this paper, we evaluate the social welfare implications of transmission investments based on equilibrium models characterizing the competitive interaction among generation firms whose decisions in generation capacity investments and production are affected by both the transmission investments and the congestion management protocols of the transmission system operator. Our analysis shows that both the magnitude of the welfare gains associated with transmission investments and the location of the best transmission expansions may change when the generation expansion response is taken into consideration. We illustrate our results using a 30-bus network example.
\end{abstract}

An incorrect version of this article was published online. The correct version is given here. The online version of the original article can be found at http://dx.doi.org/10.1007/s11149-006-9003-y

E. E. Sauma $(\varangle)$

Industrial and Systems Engineering Department

Pontificia Universidad Católica de Chile, Av. Vicuña Mackenna \# 4860,

Raúl Deves Hall, Piso 3, Macul, Santiago, Chile

e-mail: esauma@ing.puc.cl

\section{S. S. Oren}

Industrial Engineering and Operations Research Department,

University of California at Berkeley, 4141 Etcheverry Hall, Berkeley,

CA 94720, USA

e-mail: oren@ieor.berkeley.edu 
Keywords Cournot-Nash equilibrium · Market power · Mathematical program with equilibrium constraints - Network expansion planning - Power system economics · Proactive network planner

JEL Classifications $\quad$ D43 $\cdot$ L13 $\cdot$ L22 $\cdot$ L94

\section{Introduction}

Within the past decade, many countries-including the US - have restructured their electric power industries, which essentially have changed from being dominated by vertically integrated regulated monopolies (where the generation and the transmission sectors were jointly planned and operated) to deregulated industries (where generation and transmission are both planned and operated by different entities). Under the integrated monopoly structure, planning and investment in generation and transmission, as well as operating procedures, were, at least in theory, closely coordinated through an integrated resource planning (IRP) process that accounted for the complementarity and substitutability between the available resources in meeting reliability and economic objectives. ${ }^{1}$ The vertical separation of the generation and transmission sectors has resulted in a new operations and planning paradigm where IRP is no longer a viable alternative. Planning and investment in the privately owned generation sector is driven by economic considerations in response to market prices and incentives. The transmission system, on the other hand, is operated by independent transmission organizations that may or may not own the transmission assets. Whether the transmission system is owned by the system operator as in the UK or by separate owners as in some parts of the US, the transmission system operator plays a key role in assessing the needs for transmission investments from reliability and economic perspectives and in evaluating proposed investments in transmission. With few exceptions, the primary drivers for transmission upgrades and expansions are reliability considerations and interconnection of new generation facilities. ${ }^{2}$ However, because the operating and investment decisions by generation companies are market driven, valuation of transmission expansion projects must also anticipate the impact of such investments on market prices and demand response. Such economic assessments must be carefully scrutinized since market prices are influenced by a variety of factors including the ownership structure of the generation sector, the network

\footnotetext{
1 Arguably an idealized IRP was never practical for various reasons including institutional ones, which prevented or delayed various transmission projects (the California-Oregon transmission project and Devers-Palo Verde 2 project in California were cited as examples of IRP failures by one of the referees).

2 In this paper, we refer to transmission upgrades driven by reliability considerations as the transmission expansions that safely and reliably support increased demand and interconnections of new generation facilities to the grid, according to the reliability standards established by NERC, WECC, and Local Reliability Criteria.
} 
topology, the distribution and elasticity of demand, uncertainties in demand, as well as generation and network contingencies.

Existing methods for assessing the economic impact of transmission upgrades focus on the social impact of the investments, in the context of a competitive market based on locational marginal pricing (LMP), given the current generation stock. These assessments typically ignore market power effects and potential strategic response by generation investments to the transmission upgrades. For example, the Transmission Economic Assessment Methodology (TEAM) developed by the California ISO (2004) is based on the "gains from trade" principle (see (Sheffrin, 2005)), which ignores possible distortion due to market power. ${ }^{3}$

The main purpose of this paper is to introduce a methodology for assessing the economic impact of transmission investment while anticipating the strategic response of oligopolistic generation companies in generation investment and in the subsequent spot market behavior. We evaluate the social-welfare implications of transmission investments based on equilibrium models characterizing the competitive interaction among the generation firms whose decisions in generation capacity investments and production are affected by both the transmission investments and the congestion management protocols of the transmission system operator. In particular, we formulate a three-period model for studying how the exercise of local market power by generation firms affects the equilibrium between the generation and the transmission investments and, in this way, the valuation of different transmission expansion projects. In our model, we determine the social-welfare implications of transmission investments by solving a simultaneous Nash-Cournot game that characterizes the market equilibrium with respect to production quantities and prices. Our model accounts for the transmission network constraints, through a lossless DC approximation of Kirchoff's laws, as well as for demand uncertainty and for generation and transmission contingencies. Generation firms are assumed to choose their output levels at each generation node so as to maximize profits given the demand functions, the production decisions of their rivals and the import/export decisions by the system operator who is charged with maintaining network feasibility while maximizing social welfare. Assuming linear demand functions and quadratic generation cost functions the simultaneous set of Karush-Kuhn-Tucker (KKT) conditions characterizing the market equilibrium is a linear complementarity problem (LCP) for which we can compute a unique solution.

We characterize a transmission planning process whose objective is to maximize social welfare based on the valuation methodology outlined above as

\footnotetext{
3 While TEAM considers alternative generation-expansion scenarios with and without the transmission upgrades, as far as we know, this generation-expansion analysis is "open-loop" in the sense that TEAM does not take into account the potential strategic response to transmission investment from generation firms who may alter their investment plans in new generation capacity. Unlike the assumptions made in our model, two key assumptions regarding generation investment underlying TEAM are: (i) investment in new generation capacity is non-strategic and independent of transmission planning and (ii) new investment in generation capacity is just sufficient to maintain prices at levels that are competitive while providing an adequate rate of return.
} 
proactive network planning (PNP) since it accounts for the ability of a network planner to influence generation investment and the subsequent spot market behavior. We compare the social welfare implications of the PNP paradigm to two benchmark approaches:

(i) An ideal integrated-resource planner (IRP) who co-optimizes generation and transmission expansions and who dispatches generation resources and sets nodal energy spot prices so as to maximize social welfare.

(ii) A "reactive" network planner (RNP) who assumes that the generation capacities are given, thus, ignoring the interrelationship between transmission and the generation investment and optimizing the social-welfare impact of transmission expansions based only on the changes they induce in the spot market equilibrium. ${ }^{4}$

The main purpose of introducing these benchmarks is to illustrate the differences in the implied valuation of economic impact and more importantly how the different approaches may affect the optimal selection of transmission expansion and the resulting economic consequences.

Intuitively one expects that social-welfare gains under the PNP approach will be dominated by an ideal IRP but dominate the RNP. For completeness we will provide a formal proof of that intuitive relationship, which is also demonstrated numerically for a stylized 30-bus system with six generation firms.

From a regulatory policy perspective, the above observation suggests that PNP can recoup some of the welfare lost due to the unbundling of the generation and the transmission investment decisions. The implication is that society will be better served if ISO/RTOs will play a proactive role in regional transmission planning.

The concept of a proactive network planner (PNP) was formerly proposed by Craft (1999) in her doctoral thesis. However, Craft only studied the optimal network expansion in a 3-node network that presented very particular characteristics. Specifically, Craft's work assumes that only one line is congested (and only in one direction), only one node has demand, energy market is perfectly competitive, and transmission investments are not lumpy. These strong, and quite unrealistic, assumptions limit the applicability of Craft's results.

While some authors have considered the effect of the exercise of local market power on network planning, none of them have explicitly modeled the interrelationship between the transmission and the generation investment decisions. ${ }^{5}$ In Cardell, Hitt, and Hogan (1997), Joskow and Tirole (2000), Oren (1997), and Stoft (1999), the authors study the implications of market power in congested two- and/or three-node networks where the entire system demand is

\footnotetext{
4 To the extent that TEAM does not take into consideration the potential strategic response of generation firms in their capacity planning, it fits the RNP paradigm.

5 In Latorre, Cruz, Areiza, and Villegas (2003), the authors present a comprehensive list of the models on transmission expansion planning appearing in the literature. However, none of the over 100 models considered in that literature review explicitly considers the interrelationship between the transmission and the generation investment decisions.
} 
concentrated in only one node. The main idea behind these papers is that if an expensive generator with local market power is requested to produce power as result of network congestion, then the generation firm owning this generator may not have an incentive to relieve congestion. Borenstein, Bushnell, and Stoft (2000) present an analysis of the relationship between transmission capacity and generation competition in the context of a two-node network in which there is local demand at each node. The authors argue that relatively small transmission investment may yield large payoffs in terms of increased competition. Bushnell and Stoft (1996) propose to grant financial rights (which are tradable among market participants) to transmission investors as reward for the transmission capacity added to the network and suggest a transmissionrights allocation rule based on the concept of feasible dispatch. They prove that, under certain circumstances, such a rule can eliminate the incentives for a detrimental grid expansion. However, these conditions are very stringent. Joskow and Tirole (2000) analyze the Bushnell-and-Stoft's model under assumptions that better reflect the physical and economic attributes of real transmission networks. They show that a variety of potentially significant performance problems then arise.

Some other authors have proposed more radical changes to the transmission power system. Alvarado and Oren (2002) and Oren, Gross, and Alvarado (2002), for instance, propose a transmission model in which a for-profit independent transmission company (ITC) owns and operates most of its transmission resources and is responsible for operations, maintenance, and investment of the whole transmission system. Under this model, the ITC has the appropriate incentives to invest in transmission. However, the applicability of this model to actual power systems is complicated because this approach requires the divestiture and transfer to a single ITC of all transmission assets currently held by multiple owners.

Recently, Murphy and Smeers (2005) have proposed a detailed two-period model of investments in generation capacity in restructured electricity systems. In this two-stage game, generation investment decisions are made in a first stage while spot market operations occur in the second stage. Accordingly, the firststage equilibrium problem is solved subject to equilibrium constraints. However, this model does not take into consideration the transmission constraints generally present in network planning problems. Thus, since our paper focuses on the social-welfare implications of transmission investments, we make use of a simplified version of the two-period generation-capacity investment model while still solving the generation-capacity equilibrium problem as an optimization problem subject to equilibrium constraints.

The rest of this paper is organized as follows. Section 2 describes the proposed transmission investment model. In Sect. 3, we compare the valuation process of the transmission investments under the PNP paradigm with both the valuation process under integrated-resources planning (IRP) and the valuation process under the RNP paradigm. Section 4 illustrates the theoretical results presented in the previous section using a 30-bus network example. Conclusions are presented in Sect. 5. 


\begin{tabular}{|c|c|c|}
\hline Period 1 & Period 2 & Period 3 \\
\hline $\begin{array}{l}\text { The network planner } \\
\text { evaluates different } \\
\text { transmission } \\
\text { expansion projects }\end{array}$ & $\begin{array}{l}\text { Each firm invests in new } \\
\text { generation capacity, which } \\
\text { decreases its marginal cost } \\
\text { of production }\end{array}$ & $\begin{array}{l}\text { Energy } \\
\text { market } \\
\text { operation }\end{array}$ \\
\hline
\end{tabular}

Fig. 1 Three-period transmission investment valuation model

\section{The proactive transmission investment valuation model}

We introduce a three-period model for studying how generation firms' local market power affects both the firms' incentives to invest in new generation capacity and the valuation of different transmission expansion projects. The basic idea behind this model is that the interrelationship between the generation and the transmission investments affects the social value of the transmission capacity.

\subsection{Assumptions}

The model assumes a general network topology, as in a typical power-flow formulation, with possible congestion on multiple lines. To simplify the formulation, we assume, however, that all nodes are both demand and generation nodes and that all generation capacity at a node is owned by a single firm. However, firms can own generation capacity at multiple nodes. Generation firms may exercise local market power and their interaction is characterized through Cournot competition as detailed below.

The model consists of three periods, as displayed in Fig. 1. We assume that, at each period, all previous-periods actions are observable to the players who base their current decisions on that information and on their "correct" rational expectation about the behavior of all other players in the current period and on subsequent period outcomes. Thus, the proactive transmission investment valuation model is characterized as a "complete- and perfect-information" game ${ }^{6}$ and the equilibrium as "sub game perfect".

This model is static. That is, the model parameters (demand and cost functions, electric characteristics of the transmission lines, etc.) do not change over time. One way of interpreting this model is considering that it represents an investment cycle with sufficient lead time between the periods while period 3 encapsulates the average outcomes of a recurring spot energy market realization under multiple demand and supply contingencies. For each realization of demand uncertainty and transmission and generation contingencies, we compute the spot market equilibrium outcomes and calculate the average or

6 A "complete- and perfect-information" game is defined as a game in which players move sequentially and, at each point in the game, all previous actions are observable to all players. 
expected profits of the generation firms over all possible realizations. ${ }^{7}$ All the costs and benefits represented in the model are annualized.

We now explain the model backwards. The last period (period 3) represents the energy market operation. That is, in this period, we compute the equilibrium quantities and prices of electricity for given generation and transmission capacities. We model the energy market equilibrium in the topology of the transmission network through a lossless DC approximation of Kirchhoff's laws. Specifically, flows on lines are calculated using the power transfer distribution factor (PTDF) matrix, whose elements give the proportion of flow on a particular line resulting from an injection of one unit of power at any particular node and a corresponding withdrawal at an arbitrary (but fixed) slack bus. Different PTDF matrices, with corresponding state probabilities, characterize uncertainty regarding the realized network topology where the generation and transmission capacities are subject to random fluctuations, or contingencies, that are realized in period 3 prior to the production and redispatch decisions by the generation firms and the system operator. We will assume that the probabilities of all credible contingencies are public knowledge.

As in Yao, Oren, and Adler (2004), we model the energy market equilibrium as a subgame with two stages. In the first stage, Nature picks the state of the world (and, thus, settles the actual generation and transmission capacities as well as the shape of the demand and cost functions at each node). In the second stage, firms compete in a Nash-Cournot fashion by selecting their production quantities so as to maximize their profit while taking as given the production quantities of their rivals and the simultaneous import/export decisions of a system operator. The system operator determines import/export quantities at each node, taking the production decisions as given, so as to maximize social welfare while satisfying the energy balance and transmission constraints. In this setup, the production decision of the generation firms and the import/export decisions by the system operator are modeled as simultaneous moves.

In the second period, each firm invests in new generation capacity, which lowers its marginal cost of production at any output level. For the sake of tractability, we assume that generators' production decisions are not constrained by physical capacity limits. Instead, we allow generators' marginal cost curves to rise smoothly so that production quantities at any node will be limited only by economic considerations and transmission constraints. In this framework, generation expansion is modeled as "stretching" the supply function so as to lower the marginal cost at every output level and thus increase the amount of economic production at any given price. Such expansion can be interpreted as an increase in generation capacity in a way that preserves the proportional heat curve or, alternatively, assuming that any new generation capacity installed will replace old, inefficient plants and, thereby, increase the overall efficiency of the portfolio of plants in producing a given amount of electricity. This continuous representation of the supply function and generation expansion serves

7 It should be emphasized that the result of such calculations is different than the profits corresponding to an equilibrium based on expected or average demand. 
as a proxy to actual supply functions that end with a vertical segment at the physical capacity limit. Since typically generators are operated so as not to hit their capacity limits (due to high heat rates and costly wear on the generators), our proxy should be expected to produce realistic results.

The return from the generation capacity investments made in period 2 occurs in period 3, when such investments enable the firms to produce electricity at lower cost and sell more of it at a profit. We assume that, in making their investment decisions in period 2, generation firms are aware of the transmission expansion from period 1 and form rational expectations regarding the investments made by their competitors and the resulting expected market equilibrium in period 3. Thus, the generation investment and production decisions by the competing generation firms are modeled as a two- stage subgame-perfect Nash equilibrium.

Finally, in the first period, the network planner (or system operator as in some parts of the US), which we model as a Stackelberg leader in our threeperiod game, evaluates different projects to expand the transmission network while anticipating the generators' and the system operator's response in periods 2 and 3. In particular, we consider here the case where the network planner evaluates a single transmission expansion decision, but the proposed approach can be extended to more complex investment options.

Because the network planner under this paradigm anticipates the response by the generation firms, optimizing the transmission investment plan will also determine the best way of inducing generation investment so as to maximize the objective function set by the network planner (usually social welfare). Therefore, we will use the term "proactive network planner" to describe such a planning approach which results in outcomes that, although they are still inferior to the integrated-resource planning paradigm, they often result in the same investment decisions. In this model, we limit the transmission investment decisions to expanding the capacity of any one line according to some specific transmission-planning objective (the maximization of expected social welfare in this case). ${ }^{8}$ Our model allows both the upgrades of existing transmission lines and the construction of new transmission lines. Transmission upgrades that affect the electric properties of lines will obviously alter the PTDF matrices. Consequently, our model explicitly takes into consideration the changes in the PTDF matrices that are induced by alterations in either the network structure or the electric characteristics of transmission lines.

Since the energy market equilibrium will be a function of the thermal capacities of all constrained lines, the Nash equilibrium of generation capacities will also be a function of these capacity limits. The PNP, then, has multiple ways of influencing this Nash equilibrium by acting as a Stackelberg leader who anticipates the equilibrium of generation capacities and induces generation firms to make more socially optimal investments.

\footnotetext{
8 "Expected social welfare" is defined as the sum of consumer surplus, producer surplus, and congestion rent that is expected before the realization of the spot market.
} 
We further assume that the generation cost functions are both increasing and convex in the amount of output produced and decreasing and convex in the generation capacity. Furthermore, as we mentioned before, we assume that the marginal cost of production at any output level decreases as the generation capacity increases. Moreover, we assume that both the generation capacity investment cost and the transmission capacity investment cost are linear in the extra-capacity added. We also assume downward-sloping, linear demand functions at each node. To further simplify things, we assume no wheeling fees.

\subsection{Notation}

Sets:

$N$ : set of all nodes.

$L$ : set of all transmission lines.

$C$ : set of all states of contingencies.

$N_{G}$ : set of generation nodes controlled by generation firm $G$

$\Psi$ : set of all generation firms

Decision variables: ${ }^{9}$

$q_{i}^{c}$ : quantity generated at node $i$ in state $c$.

$r_{i}^{c}$ : adjustment quantity into/from node $i$ by the system operator in state $c$.

$g_{i}$ : expected generation capacity available at node $i$ after implementing the decisions made in period 2.

$f_{\ell}$ : expected thermal capacity limit of line $\ell$ after implementing the decisions made in period 1.

Parameters:

$g_{i}^{0}$ : expected generation capacity available at node $i$ before period 2 .

$f_{\ell}^{0}$ : expected thermal capacity limit of line $\ell$ before period 1 .

$g_{i}^{c}$ : generation capacity available at node $i$ in state $c$, given $g_{i}$.

$f_{\ell}^{c}$ : thermal capacity limit of line $\ell$ in state $c$, given $f_{\ell}$.

$P_{i}^{c}(\cdot)$ : inverse demand function at node $i$ in state $c$.

$\mathrm{CP}_{i}^{c}\left(q_{i}^{c}, g_{i}^{c}\right)$ : production cost function at node $i$ in state $c$.

$\mathrm{CIG}_{i}\left(g_{i}, g_{i}^{0}\right)$ : cost of investment in generation capacity at node $i$ to bring expected generation capacity to $g_{i}$.

$\mathrm{CI}_{\ell}\left(f_{\ell}, f_{\ell}^{0}\right)$ : investment cost in line $\ell$ to bring expected transmission capacity to $f_{\ell}$

$\phi_{\ell, i}^{c}(\mathrm{~L}):$ PTDF on line $\ell$ with respect to a unit injection/withdrawal at node $i$, in state $c$, when the network properties (network structure and electric characteristics of all lines) are given by the set $L$.

9 In the definition of both the decision variables and the parameters of the model, expectation is taken over the different contingent states characterizing uncertainty regarding the realization of the spot energy market. 


\subsection{Formulation}

We start by formulating the third-period problem. In the first stage of period 3, Nature determines the state of the world. In the second stage, for a given state $c$, generation firm $G(G \in \Psi)$ solves the following profit-maximization problem:

$$
\begin{aligned}
& \max _{\left\{q_{i}^{c}, i \in N_{G}\right\}} \pi_{G}^{c}=\sum_{i \in N_{G}}\left\{P_{i}^{c}\left(q_{i}^{c}+r_{i}^{c}\right) \cdot q_{i}^{c}-\mathrm{CP}_{i}^{c}\left(q_{i}^{c}, g_{i}^{c}\right)\right\} \\
& \text { s.t. } q_{i}^{c} \geq 0, \quad i \in N_{G}
\end{aligned}
$$

Simultaneously with the generators' production quantity decisions, the system operator solves the following welfare maximizing redispatch problem (for the given state $c$ ):

$$
\begin{aligned}
& \max _{\left\{r_{i}^{c}, i \in N\right\}} \Delta W^{c}=\sum_{i \in N} \int_{0}^{r_{i}^{c}} P_{i}^{c}\left(q_{i}^{c}+x_{i}\right) \mathrm{d} x_{i} \\
& \text { s.t. } \sum_{i \in N} r_{i}^{c}=0 \\
& -f_{\ell}^{c} \leq \sum_{i \in N} \phi_{\ell, i}^{c}(L) \cdot r_{i}^{c} \leq f_{\ell}^{c}, \quad \forall \ell \in L \\
& q_{i}^{c}+r_{i}^{c} \geqslant 0, \quad \forall i \in N
\end{aligned}
$$

Given that we assume no wheeling fees, the system operator can gain social surplus, at no extra cost, by exporting some units of electricity from a cheapgeneration node while importing them to other nodes until the prices at the nodes are equal, or until some transmission constraints are binding.

The previously specified model assumptions guarantee that both (1) and (2) are concave programming problems, which implies that first order necessary conditions (i.e. KKT conditions) are also sufficient. ${ }^{10}$ Consequently, to solve the period-3 problem (energy market equilibrium), we can just jointly solve the KKT conditions of the problems defined in (1), for all $G \in \Psi$, and (2), which together form a LCP that can be solved with off-the-shelf software packages.

The KKT conditions for the problems defined in (1) are:

$$
\begin{gathered}
P_{i}^{c}\left(q_{i}^{c}+r_{i}^{c}\right)+P_{i}^{c^{\prime}}\left(q_{i}^{c}+r_{i}^{c}\right) \cdot q_{i}^{c}-\frac{\partial \mathrm{CP}_{i}^{c}\left(q_{i}^{c}, g_{i}^{c}\right)}{\partial q_{i}^{c}}+\gamma_{i}^{c}=0, \quad \forall i \in N_{G}, G \in \Psi, \quad c \in C \\
\gamma_{i}^{c} \cdot q_{i}^{c}=0, \quad \forall i \in N_{G}, \quad G \in \Psi, \quad c \in C \\
q_{i}^{c} \geq 0, \quad \forall i \in N_{G}, \quad G \in \Psi, \quad c \in C \\
\gamma_{i}^{c} \geq 0, \quad \forall i \in N_{G}, \quad G \in \Psi, \quad c \in C
\end{gathered}
$$

10 An important implication of the assumptions made in Sect. 2.1 is that the problems defined in (1) and (2) are concave maximization problems over convex sets and hence yield a unique equilibrium. We recognize that these assumptions may not hold under practical circumstances (e.g. when startup costs, no load costs and minimum load constraints are taken into consideration). 
where $\gamma_{i}^{c}$ denote the Lagrange multipliers associated to the non-negativity constraints in (1).

The KKT conditions for the problem defined in (2) are:

$$
\begin{aligned}
& P_{i}^{c}\left(q_{i}^{c}+r_{i}^{c}\right)+\alpha^{c}+\sum_{\ell \in L}\left(\lambda_{\ell-}^{c}-\lambda_{\ell+}^{c}\right) \cdot \phi_{\ell, i}^{c}(L)+\beta_{i}^{c}=0, \quad \forall i \in N, \quad c \in C \\
& \sum_{i \in N} r_{i}^{c}=0, \quad \forall c \in C \\
& -f_{\ell}^{c} \leq \sum_{i \in N} \phi_{\ell, i}^{c}(L) \cdot r_{i}^{c} \leqslant f_{\ell}^{c}, \quad \forall \ell \in L, \quad c \in C \\
& q_{i}^{c}+r_{i}^{c} \geq 0, \quad \forall i \in N, c \in C \\
& \lambda_{\ell-}^{c} \cdot\left(f_{\ell}^{c}+\sum_{i \in N} \phi_{\ell, i}^{c}(L) \cdot r_{i}^{c}\right)=0, \quad \forall \ell \in L, c \in C \\
& \lambda_{\ell+}^{c} \cdot\left(f_{\ell}^{c}-\sum_{i \in N} \phi_{\ell, i}^{c}(L) \cdot r_{i}^{c}\right)=0, \quad \forall \ell \in L, c \in C \\
& \beta_{i}^{c} \cdot\left(q_{i}^{c}+r_{i}^{c}\right)=0, \quad \forall i \in N, \quad c \in C \\
& \lambda_{\ell-}^{c} \geq 0, \quad \forall \ell \in L, \quad c \in C \\
& \lambda_{\ell+}^{c} \geq 0, \quad \forall \ell \in L, c \in C \\
& \beta_{i}^{c} \geq 0, \quad \forall i \in N, c \in C
\end{aligned}
$$

where $\alpha^{c}$ is the Lagrange multiplier corresponding to the adjustment-quantities balance constraint, $\lambda_{\ell_{-}}^{c}$ and $\lambda_{\ell_{+}}^{c}$ are the Lagrange multipliers associated with the transmission capacity constraints, and $\beta_{i}^{c}$ are the Lagrange multipliers associated with the non-negativity constraints in (2).

In period 2, each firm determines how much to invest in new generation capacity by maximizing the expected value of the investment (we assume riskneutral firms) subject to (3)-(16), which represent the anticipated actions in period 3 . Since the investments in new generation capacity reduce the expected 
marginal cost of production, the return from the investments made in period 2 occurs in period 3. Thus, in period 2, each firm $G(G \in \Psi)$ solves the following optimization problem:

$$
\begin{aligned}
& \max _{\left\{g_{i}, i \in N_{G}\right\}} E_{c}\left[\pi_{G}^{c}\right]-\sum_{i \in N_{G}}\left\{\mathrm{CIG}_{i}\left(g_{i}, g_{i}^{0}\right)\right\} \\
& \text { s.t. (3)-(16) }
\end{aligned}
$$

The problem defined in (17) is a Mathematical Program with Equilibrium Constraints (MPEC) problem. ${ }^{11}$ Thus, the period-2 problem can be converted to an Equilibrium Problem with Equilibrium Constraints (EPEC), in which each firm faces (given other firms' commitments and the system operator's import/export decisions) an MPEC problem. However, this EPEC is constrained by a non-convex region and, therefore, we cannot simply write down the first order necessary conditions for each firm and aggregate them into a large problem to be solved directly. In Sect. 4, we solve this problem for the particular case-study network, using a sequential quadratic programming algorithm.

In the first period, the network planner evaluates different transmission expansion projects. In this period, the network planner is limited to deciding which line (among both the already existing lines and some proposed new lines) should be upgraded, and what should be the transmission capacity for that line, in order to maximize the expected social welfare subject to the equilibrium constraints representing the anticipated actions in periods 2 and $3 .{ }^{12}$ Thus, in period 1, the PNP's social-welfare-maximizing problem is:

$$
\max _{\ell, f_{\ell}} \sum_{i \in N}\left\{E_{c}\left[\int_{0}^{q_{i}^{c}+r_{i}^{c}} P_{i}^{c}(q) \mathrm{d} q-\mathrm{CP}_{i}^{c}\left(q_{i}^{c}, g_{i}^{c}\right)\right]-\mathrm{CIG}_{i}\left(g_{i}, g_{i}^{0}\right)\right\}-\mathrm{CI}_{\ell}\left(f_{\ell}, f_{\ell}^{0}\right)
$$

s.t. (3)-(16)

and all optimality conditions of period-2 problem.

We will not attempt to solve this problem, but rather use this formulation as a framework for evaluating alternative predetermined transmission expansion proposals. For that purpose, we will only focus on the benefit portion of the objective function in (18), which can be contrasted with the transmission investment cost. In our case study, we will only compare benefits, which is equivalent

11 For formal definitions of MPEC and EPEC problems, see (Yao et al., 2004).

12 No attempt is made here to co-optimize the network planner/system operator's transmission expansion and redispatch decisions. We assume that the transmission planning function treats the real-time redispatch function as an independent follower and anticipates its equilibrium response as if it was an independently controlled entity with no attempt to exploit possible coordination between transmission planning and real-time dispatch. One should keep in mind, however, that such coordination might be possible in a for-profit system operator enterprise such as in the UK. 
to assuming that all candidate transmission investments have the same costexcept, of course, for the "no investment" alternative which costs nothing. ${ }^{13}$

\section{Theoretical results}

In the previous section, we formulated the transmission investment valuation model used by a PNP. In this section, we present a comparative static analysis comparing the valuations of transmission investment projects from a PNP perspective with the corresponding valuations under the IRP paradigm and under the RNP paradigm. We formally show that the expected social welfare gains under the IRP and the RNP plans provide upper and lower bounds for the objective-function value corresponding to the optimal PNP plan. It should be noted, however, that the optimal decision itself may vary in an unpredictable way among the three planning paradigms. Indeed, in our numerical example (in Sect. 4), it turns out that the best investment alternative for the RNP is the same as for the IRP (although the welfare gains are different) but it is different for the PNP.

In order to facilitate the comparison, we first introduce mathematical formulations of both the IRP and the RNP transmission investment valuation models.

\subsection{Integrated-resources planner (IRP) model}

In this model, we assume that the generation and the transmission sectors are jointly planned and operated by the IRP. That is, the IRP not only jointly plans generation and transmission expansions, but it also performs the energy market operations. Thus, the IRP can incorporate the system-wide effects (operational and investment complementarities and substitutabilities between the generation and the transmission sectors), when making operations and investment decisions, in order to obtain a more socially efficient outcome. The IRP model consists of two periods: A and B. The last period (period B) corresponds to the energy market operation and it is modeled as a subgame with two stages. While Nature picks the state of the world in the first stage, the IRP chooses generation quantities and redispatch amounts (for the given state of the world) in the second stage so that social welfare is maximized. That is, the IRP solves the following social-welfare-maximizing problem (for a given state $c$ ) in period B:

13 By ignoring transmission investment costs when evaluating the economic impact of a transmission upgrade, we provide an upper limit on the economic investment costs. Obviously when comparing alternative investment plans, net benefits which account for the investment cost, should be considered. 


$$
\begin{aligned}
\max _{\left\{q_{i}^{c}, r_{i}^{c}, i \in N\right\}} & \sum_{i \in N}\left\{\int_{0}^{q_{i}^{c}+r_{i}^{c}} P_{i}^{c}(q) \mathrm{d} q-\mathrm{CP}_{i}^{c}\left(q_{i}^{c}, g_{i}^{c}\right)\right\} \\
\text { s.t. } & \sum_{i \in N} r_{i}^{c}=0 \\
& -f_{\ell}^{c} \leqslant \sum_{i \in N} \phi_{\ell, i}^{c}(L) \cdot r_{i}^{c} \leq f_{\ell}^{c}, \quad \forall \ell \in L \\
& q_{i}^{c}+r_{i}^{c} \geqslant 0, \quad \forall i \in N \\
& q_{i}^{c} \geq 0, \quad \forall i \in N
\end{aligned}
$$

In the first period (period A), the IRP jointly selects the generation investment levels and the social-welfare-maximizing location and magnitude for transmission expansion. Hence, in period A, the IRP solves the following social-welfaremaximizing problem:

$\max _{\left\{g_{i}\right\}, \ell, f_{\ell}} \sum_{i \in N}\left\{E_{c}\left[\int_{0}^{q_{i}^{c}+r_{i}^{c}} P_{i}^{c}(q) \mathrm{d} q-\mathrm{CP}_{i}^{c}\left(q_{i}^{c}, g_{i}^{c}\right)\right]-\mathrm{CIG}_{i}\left(g_{i}, g_{i}^{0}\right)\right\}-\mathrm{CI}_{\ell}\left(f_{\ell}, f_{\ell}^{0}\right)$,

s.t. all optimality conditions of period-B problem

or equivalently:

$$
\begin{aligned}
& \max _{\left\{q_{i}^{c}, r_{i}^{c}, g_{i}, \ell, f_{\ell}, i \in N\right\}} \sum_{i \in N}\left\{E_{c}\left[\int_{0}^{q_{i}^{c}+r_{i}^{c}} P_{i}^{c}(q) \mathrm{d} q-\mathrm{CP}_{i}^{c}\left(q_{i}^{c}, g_{i}^{c}\right)\right]-\mathrm{CIG}_{i}\left(g_{i}, g_{i}^{0}\right)\right\}-\mathrm{CI}_{\ell}\left(f_{\ell}, f_{\ell}^{0}\right) \\
& \text { s.t. } \sum_{i \in N} r_{i}^{c}=0, \quad \forall c \in C \\
& -f_{\ell}^{c} \leq \sum_{i \in N} \phi_{\ell, i}^{c}(L) \cdot r_{i}^{c} \leq f_{\ell}^{c}, \quad \forall \ell \in L, \forall c \in C \\
& q_{i}^{c}+r_{i}^{c} \geq 0, \quad \forall i \in N, \forall c \in C \\
& q_{i}^{c} \geq 0, \quad \forall i \in N, \quad \forall c \in C
\end{aligned}
$$

The previously specified model assumptions (ignoring non-convexities such as startup and no-load costs and minimum load constraints) guarantee that (19) is a concave programming problem, which implies that first order necessary conditions (i.e. KKT conditions) are also sufficient.

\subsection{Reactive network planner (RNP) model}

In this model, the network planner selects the social-welfare-maximizing location and magnitude for transmission upgrades assuming a fixed generation stock 
(which will typically account for firm generation investment plans), while anticipating the effect of the transmission upgrades on the energy market. This model has the same structure as the PNP model with the exception that the objective function used to evaluate alternative transmission upgrades in period 1 assumes that the generation stock upon which the energy market equilibrium is based is the current one. Thus, the third period equilibrium is again characterized by (3)-(16) with generation cost functions set based on the current generation stock. In other words, the RNP does not take into consideration the potential effect that its decisions could have on generation investment decisions in period 2 and assumes that generation capacities do not change. Thus, the RNP solves the following social-welfare-maximizing problem in the first period:

$$
\max _{\ell, f_{\ell}} \sum_{i \in N}\left\{E_{c}\left[\int_{0}^{q_{i}^{c}+r_{i}^{c}} P_{i}^{c}(q) \mathrm{d} q-\mathrm{CP}_{i}^{c}\left(q_{i}^{c}, g_{i}^{c}\right)\right]-\mathrm{CIG}_{i}\left(g_{i}, g_{i}^{0}\right)\right\}-\mathrm{CI}_{\ell}\left(f_{\ell}, f_{\ell}^{0}\right)
$$

s.t. (3) $-(16)$

$$
g_{i}=g_{i}^{0}, \quad \forall i \in N
$$

However, in evaluating the outcome of the RNP investment policy, we are considering the generation-firms' response to the transmission investment and its implication on the spot market equilibrium.

\subsection{Transmission investment valuation models comparison}

Now, we compare the optimal transmission investment decisions made for a PNP with corresponding optimal decisions of an IRP and a RNP.

Proposition 1: The optimal expected social welfare obtained from the IRP model is never smaller than the optimal expected social welfare obtained from the PNP model.

Proof By comparing (18) and (19), we observe that both problems maximize the same objective function and that all the constraints of the problem defined in (19) are included as constraints in the problem defined in (18). Moreover, the problem defined in (18) considers some other constraints. Thus, the feasible set of (18) is a subset of the feasible set of (19). Consequently, since both (18) and (19) maximize the same objective function, the optimal solution of (18) must be in the feasible set of (19), which implies that the optimal solution to (19) cannot be worse (in terms of expected social welfare) than the optimal solution of (18).

Proposition 2: The optimal expected social welfare obtained from the PNP model is never smaller than the optimal expected social welfare obtained from the RNP model. 


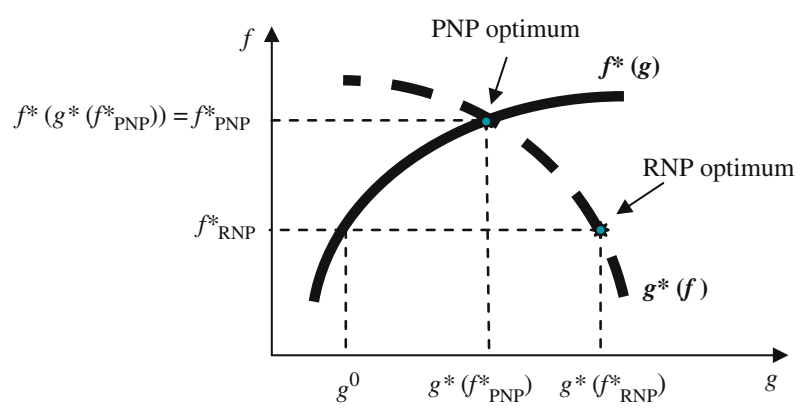

Fig. 2 Optimal transmission investments in the PNP and the RNP models

Proof By comparing (18) and (20), we observe that, if we eliminated the last constraint of each problem (second-period problem conditions), then both problems would be identical. Thus, there exists a correspondence from generation capacities space to transmission capacities space, $f^{*}(\mathrm{~g})$, that characterizes the "unconstrained" optimal investment decisions of both the PNP and the RNP. In Fig. 2, this correspondence is represented by the solid curve. ${ }^{14}$ Since the second periods of both models are identical, there also exists a correspondence from transmission capacities space to generation capacities space, $g^{*}(f)$, that characterizes the generation-firms' optimal response to transmission investments under both the PNP and the RNP models. In Fig. 2, this correspondence is represented by the dashed curve. The optimal solution of the PNP model is at the intersection of these two correspondences. That is, the transmission capacity chosen by the PNP, $f_{\mathrm{PNP}}^{*}$, is such that $f^{*}\left(g^{*}\left(f_{\mathrm{PNP}}^{*}\right)\right)=f_{\mathrm{PNP}}^{*}$. On the other hand, the transmission capacity chosen by the RNP, $f_{\mathrm{RNP}}^{*}$, is on the correspondence $f^{*}(g)$, at the currently installed generation capacities (i.e., $f_{\mathrm{RNP}}^{*}=f^{*}\left(g^{0}\right)$ ), as Fig. 2 suggests). Thus, the optimal solution of the second period of the RNP model is on the correspondence $g^{*}(f)$, at transmission capacities $f_{\mathrm{RNP}}^{*}$. Since the correspondence $g^{*}(f)$ characterizes the optimality conditions of the period2 problem in the PNP model, any pair $\left(g^{*}(f), f\right)$ represents a feasible solution of the PNP model. Consequently, the optimal solution of the RNP model, $\left(g^{*}\left(f_{\mathrm{RNP}}^{*}\right)\right.$, $f_{\mathrm{RNP}}^{*}$, is a feasible solution of the PNP model, as it is evident in Fig. 2. Therefore, the optimal solution of (18) cannot be worse (in terms of expected social welfare) than the optimal solution of (20).

Note that the previous two propositions are also valid under a different transmission-planning objective (other than expected social welfare). Consequently, we can generalize the previous propositions as in the following statement: "Under any transmission-planning objective, the optimal value obtained from the PNP model is both never larger (better) than the optimal value obtained

14 In Fig. 2, for the sake of clarity, we assume all correspondences are simple functions on the space of transmission/generation capacities. 
from the IRP model and never smaller (worse) than the optimal value obtained from the RNP model".

While Proposition 2 states that a RNP cannot do better (in terms of expected social welfare) than a PNP, the sign of the inefficiency is not evident. That is, without adding more structure to the problem, it is not evident whether the network planner underinvests or overinvests in transmission under the RNP model, relative to the PNP investment levels. To establish such comparative static results, we need a more structured characterization of the transmission investment models solutions, which requires some extra assumptions in the transmission investment models. In particular, we assume that there exist some continuous and differentiable functions that characterize the transmission investment models equilibria. This assumption is valid for small changes in transmission and generation capacities (as long as the changes do not alter the electric characteristic of the lines, i.e., as long as we can assume that the PTDFs are constant). Unfortunately, generation and transmission investments tend to be lumpy, which means that most upgrades produce large changes in generation and transmission capacities. However, the only purpose of our continuity and differentiability assumptions is to illustrate, in a simple way, that it is possible to use our 3-period transmission investment model to derive some sufficient conditions under which we can guarantee the sign of the inefficiency of the RNP model relative to the PNP model.

The optimal solution to the period-3 problem is a function of both the capacities of generators and the capacities of lines. ${ }^{15}$ Accordingly, the Nash equilibrium of generation capacities will be a function of the thermal capacities of all constrained lines. Consequently, if the cardinality of $N$ and $L$ are $n$ and $m$ respectively, and $f$ is the vector of expected thermal capacities of all the lines (i.e., $f=\left[f_{\ell 1}, \ldots, f_{\ell m}\right]^{\mathrm{T}}$ ), then we can define $g_{i}^{*}(f)$ as the period-2 Nash-equilibrium expected capacity of the generator located at node $i(i \in N), g^{*}(f)$ as the vector of all period-2 Nash-equilibrium expected generation capacities (i.e., $\left.g^{*}(f)=\left[g_{1}^{*}(f), \ldots, g_{n}^{*}(f)\right]^{\mathrm{T}}\right), q_{i}^{c *}\left(g^{*}(f), f\right)$ as the optimal quantity generated at node $i$ in state $c$ during period 3 , and $r_{i}^{c *}\left(g^{*}(f), f\right)$ as the optimal adjustment quantity into/from node $i$ by the system operator in state $c$ during period 3. As we mentioned before, we assume that $g^{*}, q_{i}^{c *}$ and $r_{i}^{c *}$ are all continuous and differentiable in all variables. ${ }^{16}$

Assuming that $q_{i}^{c *}$ and $r_{i}^{c *}$ are given continuous and differentiable functions, we can re-formulate (17) as the following unconstrained problem:

15 To be rigorous, we should say that the period-3 problem solution is also a function of both the network structure and the electric characteristics of all transmission lines.

16 In our model, $q_{i}^{c *}$ and $r_{i}^{c *}$ are continuous and differentiable in $g_{j}^{*}(\forall j \in N)$ because we assumed no upper limit in the generated quantities. To justify the continuity and differentiability with respect to $f_{l}(\forall l \in L)$, we can argue that it is possible to relax the transmission capacity constraints while introducing some adequate penalty functions into the objective function of the problem defined in (2). 


$$
\max _{\left\{g_{i}, i \in N_{G}\right\}} \sum_{i \in N_{G}}\left\{E_{c}\left[P_{i}^{c}\left(q_{i}^{c *}+r_{i}^{c *}\right) \cdot q_{i}^{c *}-\mathrm{CP}_{i}^{c}\left(q_{i}^{c *}, g_{i}^{c}\right)\right]-\mathrm{CIG}_{i}\left(g_{i} g_{i}^{0}\right)\right\}
$$

Then, for given functions $q_{i}^{c *}$ and $r_{i}^{c *}$, we can write the KKT conditions for the problems defined in (21), $\forall i \in N_{G}$, as:

$$
\begin{aligned}
\sum_{j \in N_{G}}\left\{E _ { c } \left[P_{j}^{c}\left(q_{j}^{c *}+r_{j}^{c *}\right) \cdot \frac{\partial q_{j}^{c *}}{\partial g_{i}}+P_{j}^{c \prime}\left(q_{j}^{c *}+r_{j}^{c *}\right) \cdot \frac{\partial\left(q_{j}^{c *}+r_{j}^{c *}\right)}{\partial g_{i}} \cdot q_{j}^{c *}\right.\right. \\
\left.\left.\quad-\frac{\partial \mathrm{CP}_{j}^{c}\left(q_{j}^{c *}, g_{j}^{c}\right)}{\partial q_{j}^{c *}} \cdot \frac{\partial q_{j}^{c *}}{\partial g_{i}}\right]\right\}-E_{\mathrm{c}}\left[\frac{\partial \mathrm{CP}_{i}^{c}\left(q_{i}^{c *}, g_{i}^{c}\right)}{\partial g_{i}}\right]-\frac{\partial \mathrm{CIG}_{i}\left(g_{i}, g_{i}^{0}\right)}{\partial g_{i}}=0
\end{aligned}
$$

Equation (22) indicates that the value of additional generation capacity to a firm depends on the capacity levels of all the other generators and the transmission capacities of all lines in the network. Moreover, (22) means that, under the existence of local market power, generation firms will not invest so as to just equate the expected reduction in the marginal cost of production and the marginal investment cost (i.e., the last two terms in (22)). On the contrary, firms will invest in generation capacity taking into account the effect of their investments on the energy market equilibrium.

Assuming $g^{*}, q_{i}^{c *}$ and $r_{i}^{c *}$ are given functions, we can also re-formulate (18) as the following unconstrained problem:

$$
\max _{\ell, f_{\ell}} \sum_{i \in N}\left\{E_{c}\left[\int_{0}^{q_{i}^{c *}+r_{i}^{c *}} P_{i}^{c}(q) \mathrm{d} q-\mathrm{CP}_{i}^{c}\left(q_{i}^{c *}, g_{i}^{c *}\right)\right]-\mathrm{CIG}_{i}\left(g_{i}^{*}, g_{i}^{0}\right)\right\}-\mathrm{CI}_{\ell}\left(f_{\ell} f_{\ell}^{0}\right)
$$

As indicated earlier, we do not attempt to solve this problem algorithmically, but use it as a framework for evaluating alternative investment options and compare the theoretical outcome of the three planning paradigms considered in this paper.

For given functions $g^{*}, q_{i}^{c *}$ and $r_{i}^{c *}$, we can write the KKT conditions for the problem defined in (23), for some optimal $\ell \in L$, as:

$$
\begin{gathered}
\sum_{i \in N}\left\{E _ { c } \left[P_{i}^{c}\left(q_{i}^{c *}+r_{i}^{c *}\right) \cdot\left(\sum_{j \in N}\left(\frac{\partial q_{i}^{c *}}{\partial g_{j}^{*}} \cdot \frac{\partial g_{j}^{*}}{\partial f_{\ell}}\right)+\frac{\partial q_{i}^{c *}}{\partial f_{\ell}}+\sum_{j \in N}\left(\frac{\partial r_{i}^{c *}}{\partial g_{j}^{*}} \cdot \frac{\partial g_{j}^{*}}{\partial f_{\ell}}\right)+\frac{\partial r_{i}^{c *}}{\partial f_{\ell}}\right)\right.\right. \\
\left.-\frac{\partial \mathrm{CP}_{i}^{c}\left(q_{i}^{c *}, g_{i}^{c *}\right)}{\partial q_{i}^{c *}} \cdot\left(\sum_{j \in N}\left(\frac{\partial q_{i}^{c *}}{\partial g_{j}^{*}} \cdot \frac{\partial g_{j}^{*}}{\partial f_{\ell}}\right)+\frac{\partial q_{i}^{c *}}{\partial f_{\ell}}\right)-\frac{\partial \mathrm{CP}_{i}^{c}\left(q_{i}^{c *}, g_{i}^{c *}\right)}{\partial g_{i}^{*}} \cdot \frac{\partial g_{i}^{*}}{\partial f_{\ell}}\right] \\
\left.-\frac{\partial \mathrm{CIG}_{i}\left(g_{i}^{*}, g_{i}^{0}\right)}{\partial g_{i}^{*}} \cdot \frac{\partial g_{i}^{*}}{\partial f_{\ell}}\right\}-\frac{\partial \mathrm{Cl}_{\ell}\left(f_{\ell}, f_{\ell}^{0}\right)}{\partial f_{\ell}}=0
\end{gathered}
$$


Now, we are able to establish some sufficient conditions under which we can guarantee the sign of the RNP's inefficiency in transmission investments as compared with the investment levels under the PNP model.

Proposition 3: Suppose that the gain in expected welfare of an incremental unit of generation capacity is greater (smaller) than the marginal investment cost for those generation firms whose generation capacities are strategic complements (substitutes) to the transmission capacity at the optimal location of the transmission upgrade. Furthermore, assume that the optimal location of the transmission upgrade is the same under both the RNP model and the PNP model. Then, the $R N P$ will underinvest in transmission capacity as compared to the PNP.

Proof Let $q_{i}^{c *}$ and $r_{i}^{c *}(\forall i \in N, c \in C)$ define an optimal solution for the third period of the RNP model. Then, for given functions $q_{i}^{c *}$ and $r_{i}^{c *}(\forall i \in N, c \in C)$, any optimal solution to the first-period optimization of the RNP model must satisfy the following first order optimality condition (for some optimal $\ell \in L$ ):

$$
\sum_{i \in N}\left(\partial E_{c}\left[\int_{0}^{q_{i}^{c^{*}}+r_{i}^{c^{*}}} P_{i}^{c}(q) \mathrm{d} q-\mathrm{CP}_{i}^{c}\left(q_{i}^{c *}, g_{i}^{c}\right)\right] / \partial f_{\ell}\right)-\frac{\partial \mathrm{CI}_{\ell}\left(f_{\ell}, f_{\ell}^{0}\right)}{\partial f_{\ell}}=0
$$

Then, we can re-write (24), which corresponds to the first order optimality condition of the period-1 problem of the PNP model, as follows (assuming that the optimal location of the next transmission upgrade, $\ell(\ell \in L)$, is the same under both the RNP model and the PNP model):

$$
\begin{aligned}
& \overbrace{\sum_{i \in N}\left(\partial E_{c}\left[\int_{0}^{q_{i}^{c *}+r_{i}^{c *}} P_{i}^{c}(q) \mathrm{d} q-\mathrm{CP}_{i}^{c}\left(q_{i}^{c *}, g_{i}^{c}\right)\right] / \partial f_{\ell}\right)-\frac{\partial \mathrm{CI}_{\ell}\left(f_{\ell}, f_{\ell}^{0}\right)}{\partial f_{\ell}}}^{\text {Reactivenetworkplanner }} \\
& +\underbrace{\sum_{j \in N}\left\{\sum_{i \in N}\left(\partial E_{c}\left[\int_{0}^{q_{i}^{c *}+r_{i}^{c *}} P_{i}^{c}(q) \mathrm{d} q-\mathrm{CP}_{i}^{c}\left(q_{i}^{c *}, g_{i}^{c}\right)\right] / \partial g_{j}^{*}-\frac{\partial \mathrm{CIG}_{i}\left(g_{i}^{*}, g_{i}^{0}\right)}{\partial g_{j}^{*}}\right) \cdot \frac{\partial g_{j}^{*}}{\partial f_{\ell}}\right\}}_{\text {Period-2 NE enhancenment effect by the PNP }}=0
\end{aligned}
$$

The previous optimality condition undoubtedly shows that the PNP alters its actions (as compared to an RNP) in order to recapture some of the social welfare lost due to the socially inefficient generation capacity investments.

Using (25), it follows directly from the proposition assumptions that the "period-2 Nash-equilibrium enhancement effect" made by the PNP is positive, which implies that the RNP will underinvest in transmission capacity as compared to the PNP (assuming that the optimal location of the next transmission upgrade is the same under both the RNP model and the PNP model).

Equation (25) reflects how the PNP differs from its reactive counterpart (i.e., from a network planner that ignores the dependency of the equilibrium of 
generation capacities on the transmission capacities). In addition to the welfare gained directly by adding transmission capacity, which by definition corresponds to the sum of all the shadow prices of the transmission constraints, the PNP also considers how its investment can induce a more socially efficient Nash equilibrium of expected generation capacities. In fact, it includes a "period-2 Nash-equilibrium enhancement effect" into the social value of transmission capacity. Although the sign of this effect is not evident in general, proposition-3 assumptions guarantee a positive sign.

The three previous propositions deal with the transmission investment decisions made under the different paradigms. It is also interesting to analyze what can be said about the generation investment decisions made under the corresponding models. The next proposition sets up some sufficient conditions under which we can guarantee that a generation firm will underinvest under either the PNP or the RNP paradigm as compared to investment levels implied by the IRP.

Proposition 4: Under either the PNP model or the RNP model, if a firm owning generation capacity at a single node expands its capacity such that its expected marginal profit is smaller than the expected marginal welfare gain corresponding to the IRP's optimal decision, then the generation firm underinvests relative to the IRP's optimal investment level at that node.

Proof Let $q_{i}^{\mathrm{cIRP}}$ and $r_{i}^{\mathrm{cIRP}}$ define the market equilibrium of period B in the IRP model. Then, given $q_{i}^{c \mathrm{IRP}}$ and $r_{i}^{c \mathrm{IRP}}$, any optimal solution of period A of the IRP model must satisfy the following first order optimality condition:

$$
\begin{aligned}
E_{c} & {\left[\sum_{j \in N}\left\{P_{j}^{c}\left(q_{j}^{c \mathrm{IRP}}+r_{j}^{c \mathrm{IRP}}\right) \cdot \frac{\partial\left(q_{j}^{c \mathrm{IRP}}+r_{j}^{c \mathrm{IRP}}\right)}{\partial g_{i}}-\frac{\partial \mathrm{CP}_{j}^{c}\left(q_{j}^{c \mathrm{IRP}}, g_{j}^{c}\right)}{\partial q_{j}^{c \mathrm{RPP}}} \cdot \frac{\partial q_{j}^{c \mathrm{IRP}}}{\partial g_{i}}\right\}\right] } \\
& -E_{c}\left[\frac{\partial \mathrm{CP}_{i}^{c}\left(q_{i}^{c \mathrm{IRP}}, g_{i}^{c}\right)}{\partial g_{i}}\right]-\frac{\partial \mathrm{CIG}_{i}\left(g_{i}, g_{i}^{0}\right)}{\partial g_{i}}=0
\end{aligned}
$$

On the other hand, (22) represents an optimality condition for the generationfirms' investment problem under both the PNP model and the RNP model. By comparing (22) and (26) in the case of a firm owning only the generation capacity at node $i$, it is clear that it suffices to prove that:

$$
\begin{aligned}
E_{c} & {\left[\frac{\partial\left(P_{i}^{c}\left(q_{i}^{c *}+r_{i}^{c *}\right) \cdot q_{i}^{c *}-\mathrm{CP}_{j}^{c}\left(q_{j}^{c *}, g_{j}^{c}\right)\right)}{\partial g_{i}}\right] } \\
& <\sum_{j \in N}\left\{E_{c}\left[\partial\left(\int_{0}^{q_{j}^{c \mathrm{RP}}+r_{j}^{c \mathrm{IRP}}} P_{j}^{c}(q) \mathrm{d} q-\mathrm{CP}_{j}^{c}\left(q_{j}^{c \mathrm{IRP}}, g_{j}^{c}\right)\right) / \partial g_{i}\right]\right\}
\end{aligned}
$$

The assumptions of proposition 4 directly guarantee the validity of (27). 


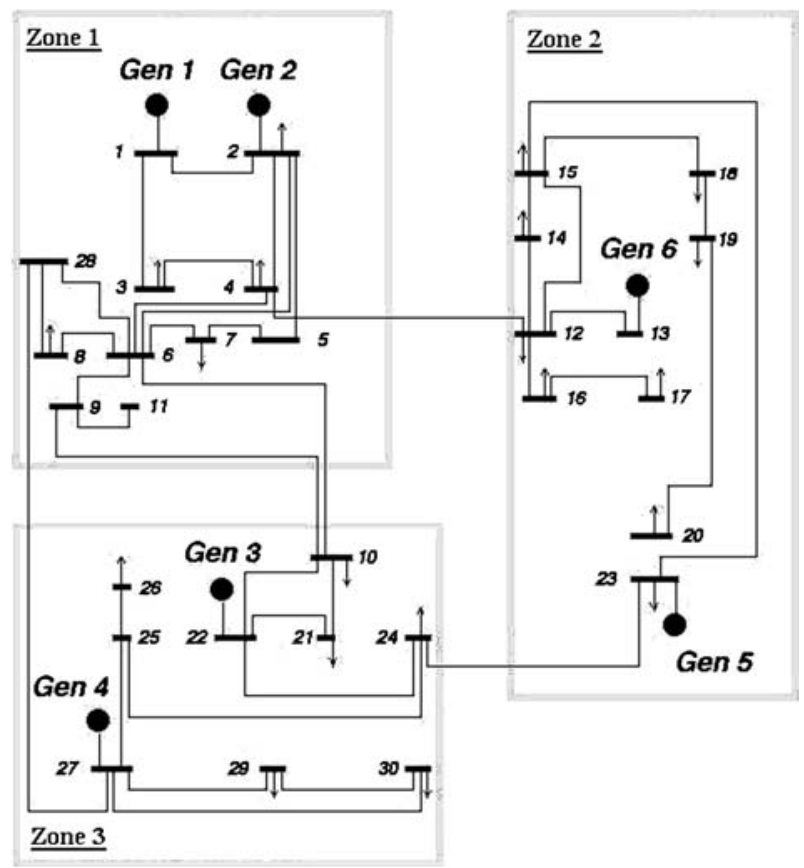

Fig. 3 30-bus Cornell network

\section{Case study}

We illustrate the theoretical results derived in the previous section using a stylized version of the 30-bus/3-zone network displayed in Fig. 3, which was developed at Cornell University for experimental economic studies of electricity markets (http://www.pserc.cornell.edu/powerweb). There are six generation firms in the market (each one owning the generation capacity at a single node). Nodes 1, 2, 13, 22, 23, and 27 are the generation nodes. There are 39 transmission lines. The electric characteristics of the transmission lines are listed in Table 7 in the Appendix.

The uncertainty associated with the energy market operation is classified into seven independent contingent states (see Table 1). Six of them have small independent probabilities of occurrence (two involve demand uncertainty, two involve network uncertainty and the other two involve generation uncertainty). Table 2 shows the nodal information in the normal state.

We assume the same production cost function, $\mathrm{CP}_{i}^{c}(\cdot)$, for all generators and all contingencies. Note that $\mathrm{CP}_{i}^{c}(\cdot)$ is increasing in $q_{i}^{c}$, but it is decreasing in $g_{i}^{c}$. Moreover, recall that we have assumed that generators have unbounded capacity (i.e., they never reach the upper generation capacity limit). Thus, the only important effect of investing in generation capacity is lowering the production cost. Moreover, we assume that all generation firms have the same investment cost function, given by $\mathrm{CIG}_{i}\left(g_{i}, g_{i}^{0}\right)=8 \cdot\left(g_{i}-g_{i}^{0}\right)$, in dollars. The before-period- 2 
Table 1 States of contingencies associated with the energy market operation

\begin{tabular}{lll}
\hline State & Probability & Type of uncertainty and description \\
\hline 1 & 0.82 & Normal state: Data set as in Table 2 \\
2 & 0.03 & Demand uncertainty: All demands increase by $10 \%$ \\
3 & 0.03 & Demand uncertainty: All demands decrease by $10 \%$ \\
4 & 0.03 & Network uncertainty: Line 15-23 goes down \\
5 & 0.03 & Network uncertainty: Line 23-24 goes down \\
6 & 0.03 & Generation uncertainty: Generator at node 1 goes down \\
7 & 0.03 & Generation uncertainty: Generator at node 13 goes down \\
\hline
\end{tabular}

Table 2 Nodal information used in the 30-bus Cornell network in the normal state

\begin{tabular}{lll}
\hline Data type (units) & Information & Nodes where it applies \\
\hline Inverse demand function $(\$ / \mathrm{MWh})$ & $P_{i}(q)=50-q$ & $1,2,5,6,9,11,13,16,18,20,21$, \\
& & $22,25,26,27,28$, and 29 \\
Inverse demand function $(\$ / \mathrm{MWh})$ & $P_{i}(q)=55-q$ & $4,8,10,12,14,15,17,19,24$, and \\
& & 30 \\
Inverse demand function $(\$ / \mathrm{MWh})$ & $P_{i}(q)=60-q$ & 3,7, and 23 \\
Generation cost function $(\$ \mathrm{MWh})$ & $\mathrm{CP}_{i}\left(q_{i}, g_{i}\right)=\left(0.25 \cdot q_{i}^{2}\right.$ & $1,2,13,22,23$, and 27 (all gener- \\
& $\left.+20 \cdot q_{i}\right) \cdot\left(g_{i}^{0} / g_{i}\right)$ & ation nodes) \\
\hline
\end{tabular}

expected generation capacity is assumed to be the same for all generation nodes and equal to $60 \mathrm{MW}$ (i.e., $g_{i}^{0}=60 \mathrm{MW} \forall i \in 1,2,13,22,23,27$ ). For our purposes, the choice of the parameter $g_{i}^{0}$ is not important because the focus of this paper is not generation adequacy. Instead, what we are really interested in is the ratio $\left(g_{i}^{0} / g_{i}\right)$ since we focus on the cost of generating power and the effect that both generation and transmission investments have over that cost.

As mentioned before, the KKT conditions of the period-3 problem of the PNP model constitute a LCP. We solve it, for each contingent state, by minimizing the complementarity conditions subject to the linear equality constraints and the non-negativity constraints. ${ }^{17}$ The period- 2 problem of the PNP model is an EPEC, in which each firm faces a Mathematical Program subject to Equilibrium Constraints (MPEC). ${ }^{18}$ We attempt to solve for equilibrium, if at least one exists, by iterative deletion of dominated strategies. That is, we sequentially solve each firm's profit-maximization problem using as data the optimal values from previously solved problems. Thus, starting from a feasible solution, we solve for $g_{1}$ using $g_{(-1)}$ as data in the first firm's optimization problem (where $g_{(-1)}$ means all firms' generation capacities except for firm 1's), then solve for

17 Recall that any LCP can be written as the problem of finding a vector $x \in \Re^{n}$ such that $x=q+M \cdot y, x^{\mathrm{T}} \cdot y=0, x \geq 0$, and $y \geq 0$, where $M \in \mathfrak{R}^{n \times n}, q \in \mathfrak{R}^{n}$, and $y \in \mathfrak{R}^{n}$. Thus, we can solve it by minimizing $x^{\mathrm{T}} \cdot y$ subject to $x=q+M \cdot y, x \geq 0$, and $y \geq 0$. If the previous problem has an optimal solution where the objective function is zero, then that solution also solves the corresponding LCP. Greater details about the methodology used for solving LCPs are given in (Hobbs, 2001).

18 See (Yao et al., 2004) for definitions of both EPEC and MPEC. 
Table 3 Generation quantities, adjustment quantities, and nodal prices in the normal state, in the PNP model, under absence of transmission investments

\begin{tabular}{llll}
\hline Node & $q_{i}(\mathrm{MWh})$ & $r_{i}(\mathrm{MWh})$ & $P_{i}(\$ / \mathrm{MWh})$ \\
\hline 1 & 27.397 & -24.827 & 47.43 \\
2 & 27.808 & -25.230 & 47.42 \\
3 & 0 & 12.544 & 47.46 \\
4 & 0 & 7.539 & 47.46 \\
5 & 0 & 2.600 & 47.40 \\
6 & 0 & 2.624 & 47.38 \\
7 & 0 & 12.614 & 47.39 \\
8 & 0 & 7.630 & 47.37 \\
9 & 0 & 2.838 & 47.16 \\
10 & 0 & 7.950 & 47.05 \\
11 & 0 & 2.838 & 47.16 \\
12 & 0 & 6.932 & 48.07 \\
13 & 24.706 & -21.547 & 46.84 \\
14 & 0 & 6.799 & 48.20 \\
15 & 0 & 6.612 & 48.39 \\
16 & 0 & 1.932 & 48.07 \\
17 & 0 & 6.932 & 48.07 \\
18 & 0 & 1.022 & 48.98 \\
19 & 0 & 6.022 & 48.98 \\
20 & 0 & 1.022 & 48.98 \\
21 & 0 & 3.033 & 46.97 \\
22 & 27.055 & -23.997 & 46.94 \\
23 & 21.724 & -7.474 & 45.75 \\
24 & 0 & 8.474 & 46.53 \\
25 & 0 & 3.152 & 46.85 \\
26 & 0 & 3.152 & 46.85 \\
27 & 26.310 & -23.354 & 47.04 \\
28 & 0 & 2.663 & 47.34 \\
29 & 0 & 2.500 & 47.50 \\
30 & 0 & 7.007 & 48.00 \\
\hline
\end{tabular}

$g_{2}$ using $g_{(-2)}$ as data, and so on. We solve each firm's profit-maximization problem using a sequential quadratic programming algorithm implemented in MATLAB ${ }^{\circledR}$.

We test our model from a set of different starting points and using different generation-firms' optimization order. All these trials gave us the same results. For the PNP model, the optimal levels of generation capacity under absence of transmission investments are $\left(g_{1}^{*}, g_{2}^{*}, g_{3}^{*}, g_{4}^{*}, g_{5}^{*}, g_{6}^{*}\right)=(100.92,103.72,101.15$, 95.94, 77.07, 87.69) in MW. Table 3 lists the corresponding generation quantities $\left(q_{i}\right)$, adjustment quantities $\left(r_{i}\right)$ and nodal prices $\left(P_{i}\right)$ in the normal state. Figure 4 illustrates these results for the Cornell network. In Fig. 4, thick lines represent the transmission lines reaching their thermal capacities (in the indicated direction) and circles are located in the nodes with the highest prices (above \$48/MWh).

To evaluate the period- 1 objective-function value corresponding to a transmission line expansion in the PNP model, we solve a period-2 problem that considers the new network data to solve the energy market equilibrium at 


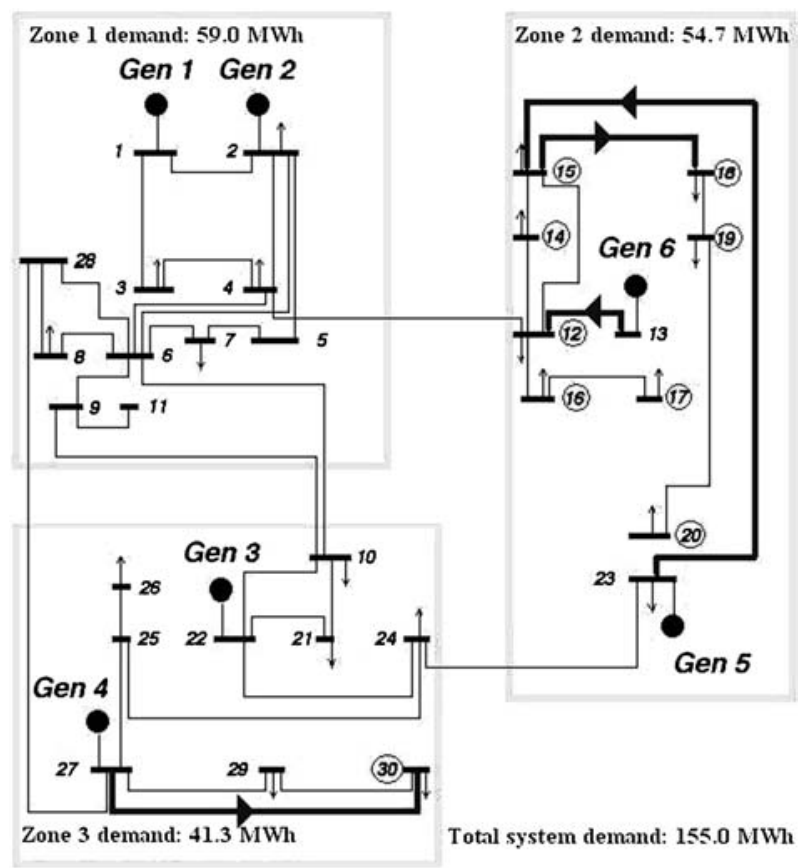

Fig. 4 Results of the PNP model in the normal state, in the absence of transmission investment, for the 30-bus Cornell network

period 3. We then compare the values obtained for alternative line expansions and identify the one producing the highest expected social welfare gain. For simplicity, we do not consider transmission investment costs. Thus, the values obtained establish upper limits on the economic investment in each line expansion (not accounting for reliability considerations).

The four congested lines in the normal state, in absence of transmission investment, are obvious candidates for the single-line upgrade. We tested the PNP decision by comparing the results of independently adding 100 MVA of capacity to each one of these four lines and to four new lines. ${ }^{19}$ The results are summarized in Table 4. In assessing the economic impacts of the alternative line expansions, we compare social-welfare implications along with the impact on market power (measured by an average Lerner index ${ }^{20}$ ), producer and

19 For simplicity, in the case of upgrading an existing line, we assume that the upgrade does not alter the electric characteristics, but only the thermal capacity of the line (for instance, this would be the case if, for the expanded line, we replaced all the wires by "low sag wires" while using the same existing high-voltage towers). On the other hand, in the case of building a line at a new location, we consider that the PTDF matrices change according to both the new network structure and the electric characteristics of the new line. For all new-line expansion projects, we evaluate the impact of the construction of a transmission line with thermal capacity equal to $100 \mathrm{MVA}$, resistance equal to 0.01 p.u., and reactance equal to 0.04 p.u.

20 The Lerner Index is defined as the fractional price markup i.e. (Price - Marginal cost)/Price. 


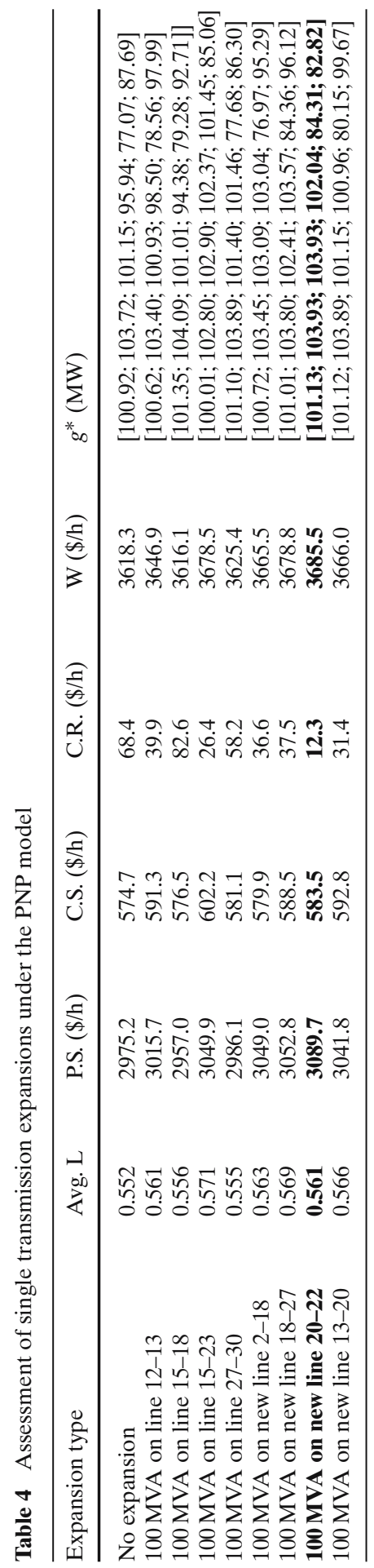


Table 5 Assessment of single transmission expansions under the RNP model

\begin{tabular}{llllll}
\hline Expansion type & $\overline{\text { Avg.L }}$ & $\overline{\text { P.S. }(\$ / h)}$ & $\overline{\text { C.S. }(\$ / h)}$ & $\overline{\text { C.R. }(\$ / h)}$ & $\overline{\mathrm{W}}(\$ / \mathrm{h})$ \\
\hline No expansion & 0.395 & 2732.4 & 387.9 & 9.1 & 3129.4 \\
100 MVA on line 12-13 & 0.395 & 2732.4 & 388.3 & 8.9 & 3129.6 \\
100 MVA on line 15-18 & 0.395 & 2732.1 & 388.3 & 8.9 & 3129.3 \\
100 MVA on line 15-23 & 0.395 & 2732.5 & 388.2 & 8.8 & 3129.5 \\
100 MVA on line 27-30 & 0.395 & 2732.4 & 387.9 & 9.1 & 3129.4 \\
100 MVA on new line 2-18 & 0.396 & 2750.4 & 386.8 & 0.5 & 3137.7 \\
100 MVA on new line 18-27 & $\mathbf{0 . 3 9 6}$ & $\mathbf{2 7 5 1 . 0}$ & $\mathbf{3 8 6 . 8}$ & $\mathbf{0 . 2}$ & $\mathbf{3 1 3 8 . 0}$ \\
100 MVA on new line 20-22 & 0.396 & 2750.7 & 386.8 & 0.3 & 3137.8 \\
100 MVA on new line 13-20 & 0.395 & 2742.6 & 387.2 & 4.3 & 3134.1 \\
\hline
\end{tabular}

consumer surplus as well as congestion rents. In Table 4, "Avg. L" corresponds to the expected Lerner index averaged over all generation firms, "P.S." is the expected producer surplus of the system, "C.S." is the expected consumer surplus of the system, "C.R." represents the expected congestion rents over the entire system, "W" is the expected social welfare of the system, and " $g^{*}$ " corresponds to the vector of all Nash-equilibrium expected generation capacities.

From Table 4, it is evident that the highest valued (in terms of expected social welfare) single transmission line expansion is a new line connecting nodes 20 and 22. Moreover, it is interesting to observe that some expansion projects (as adding 100 MVA on line 15-18) can decrease social welfare.

Now, we are interested in comparing the PNP "best expansion" with that obtained under the RNP paradigm for the same system conditions. We tested the RNP decision by comparing the results of independently adding 100 MVA of capacity to each one of the same (existing and new) eight lines as before. The results are summarized in Table 5, where we use the notation $\bar{x}$ to represent the value of $x$ as seen by the RNP.

From Table 5, it is clear that the social-welfare-maximizing transmission expansion for the RNP is, in this case, a new transmission line connecting nodes 18 and 27. In evaluating the "true outcome" corresponding to the RNP best choice, we do take into consideration the generation investment response to that "suboptimal" choice and the subsequent energy market equilibrium, which result in Avg. $\mathrm{L}=0.569$, P.S. $=\$ 3,052.8 / \mathrm{h}$, C.S. $=\$ 588.5 / \mathrm{h}$, C.R. $=\$ 37.5 / \mathrm{h}, \mathrm{W}=$ $\$ 3,678.8 / \mathrm{h}$, and $g^{*}=(101.01,103.80,102.41,103.57,84.36,96.12)$ in MW. By comparing Tables 4 and 5, it is evident that the optimal investment decision under the PNP paradigm differs from the optimal investment decision corresponding to the RNP. Specifically, the PNP considers not only the welfare gained directly by adding transmission capacity (on which the RNP bases its valuations), but also the way in which its investment induces a more socially efficient Nash equilibrium of expected generation capacities.

Finally, it is interesting to compare the results obtained with the PNP model and those obtained with an hypothetical IRP. We tested the IRP decisions by comparing the results of independently adding 100 MVA of capacity to each one of the same eight lines as before. The results are summarized in Table 6 . 


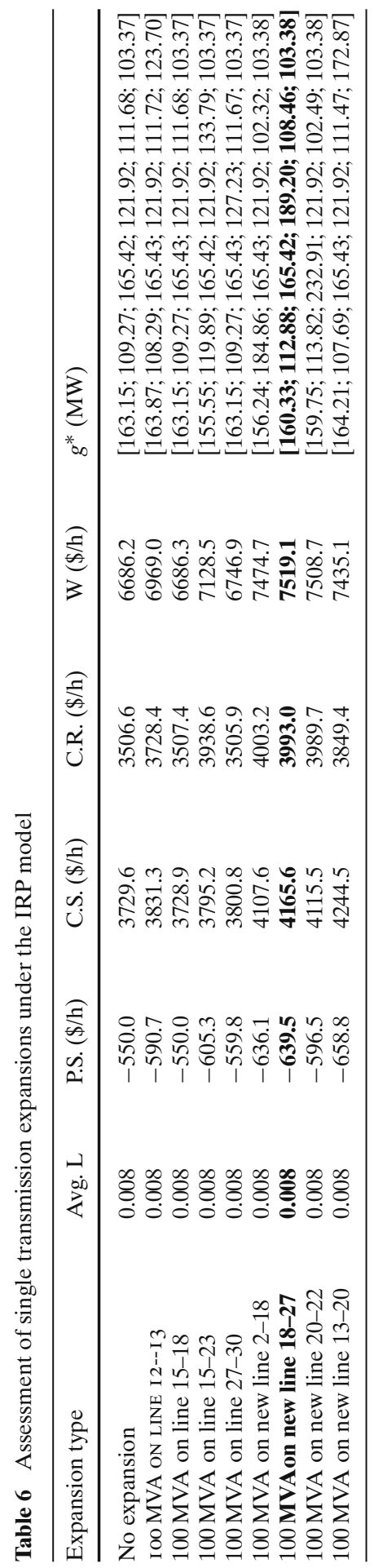


From Table 6, it is clear that the social-welfare-maximizing transmission expansion for the IRP is, in this case, a new line connecting nodes 18 and 27. By comparing Tables 4, 5 and 6 , we observe that the IRP is able to significantly increase the expected social welfare with respect to the PNP and the RNP. This is because the IRP not only jointly plans generation and transmission expansions, but it also chooses generation quantities and redispatch amounts in order to maximize the expected social welfare of the whole system. It is also interesting to note that the producer surplus under every expansion project studied here is negative due to the fact that the IRP controls all the decision variables.

\section{Conclusions}

In this paper, we evaluated the social welfare implications of transmission investments based on equilibrium models characterizing the competitive interaction among generation firms whose decisions in generation capacity investments and production are affected by both the transmission investments and the congestion management protocols of the transmission network planner. In particular, we proposed a three-period model for studying how the exercise of local market power by generation firms affects the equilibrium between the generation and the transmission investments and, in this way, the valuation of different transmission expansion projects. We showed that, although a PNP cannot do better (in terms of expected social welfare) than an IRP, it can recoup some of the lost welfare by identifying transmission investment options that are ex-post optimal given the strategic investment response by generation companies. We also proved that a RNP cannot do better (in terms of expected social welfare) than the PNP. Moreover, we illustrated through a numerical example that the valuations of transmission investments under the RNP paradigm can result in the selection of transmission expansion options that are inferior to those selected based on the PNP valuation, given the generation investment response to such expansions. Indeed, the PNP valuation methodology can identify more socially efficient expansion options than the RNP because it takes into consideration not only the welfare gained directly by adding transmission capacity, but also the way in which the transmission investment alters the Nash equilibria of expected generation capacities.

While the PNP paradigm is still inferior to IRP, which co-optimizes transmission and generation expansion, the reality is that IRP is no longer a relevant methodology in a system where generators are privately owned and investment decisions in generation are not centrally coordinated and arguably was never practical due to institutional barriers that prevailed in the vertically integrated utility regime. On the other hand, the PNP paradigm can be readily implemented as part of an economic assessment methodology employed by system operators. Such implementation requires, however, that transmission planning be centralized in a regional organization such as an ISO, an RTO or a regional performance-based-regulated ITC who will take the lead in identifying investment priorities. In doing so, the planner is able to respond to current 
needs and also guide future generation investment in the public interest. This view is contrary to the prevailing generation-centric view that gives priority to market-driven generation investment decisions and to merchant transmission investments while the ISO/RTO plays a passive (reactive) role. In this role the ISO/RTO evaluates transmission investment proposals as they come in and supplements them as needed to meet reliability requirements dictated by load growth and new generation investments. Unfortunately, this reactive approach is likely to lead to piece mill investment in the transmission infrastructure that is likely to be socially suboptimal in the long run.

Acknowledgements The authors thank R. Thomas for providing the 30-bus Cornell network data used in our case study. The work reported in this paper was supported by NSF Grant ECS011930, The Power System Engineering Research Center (PSERC) and by the Center for Electric Reliability Technology Solutions (CERTS) through a grant from the Department of Energy.

\section{Appendix}

The network data used in our case study are provided here. Table 7 lists the electric characteristics of the 39 transmission lines of the Cornell network.

Table 7 Electric characteristics of the transmission lines of the 30-bus network

\begin{tabular}{|c|c|c|c|c|c|}
\hline Line \# & From node \# & To node \# & Resistance (p.u.) & Reactance (p.u.) & $f_{\ell}^{0}$ (MVA) \\
\hline 1 & 1 & 2 & 0.02 & 0.06 & 130 \\
\hline 2 & 1 & 3 & 0.05 & 0.19 & 130 \\
\hline 3 & 2 & 4 & 0.06 & 0.17 & 65 \\
\hline 4 & 3 & 4 & 0.01 & 0.04 & 130 \\
\hline 5 & 2 & 5 & 0.05 & 0.20 & 130 \\
\hline 6 & 2 & 6 & 0.06 & 0.18 & 65 \\
\hline 7 & 4 & 6 & 0.01 & 0.04 & 90 \\
\hline 8 & 5 & 7 & 0.05 & 0.12 & 70 \\
\hline 9 & 6 & 7 & 0.03 & 0.08 & 130 \\
\hline 10 & 6 & 8 & 0.01 & 0.04 & 32 \\
\hline 11 & 6 & 9 & 0.00 & 0.21 & 65 \\
\hline 12 & 6 & 10 & 0.00 & 0.56 & 32 \\
\hline 13 & 9 & 11 & 0.00 & 0.21 & 65 \\
\hline 14 & 9 & 10 & 0.00 & 0.11 & 65 \\
\hline 15 & 4 & 12 & 0.00 & 0.26 & 65 \\
\hline 16 & 12 & 13 & 0.00 & 0.14 & 65 \\
\hline 17 & 12 & 14 & 0.12 & 0.26 & 32 \\
\hline 18 & 12 & 15 & 0.07 & 0.13 & 32 \\
\hline 19 & 12 & 16 & 0.09 & 0.20 & 32 \\
\hline 20 & 14 & 15 & 0.22 & 0.20 & 16 \\
\hline 21 & 16 & 17 & 0.08 & 0.19 & 16 \\
\hline 22 & 15 & 18 & 0.11 & 0.22 & 16 \\
\hline 23 & 18 & 19 & 0.06 & 0.13 & 16 \\
\hline 24 & 19 & 20 & 0.03 & 0.07 & 32 \\
\hline 25 & 10 & 21 & 0.03 & 0.07 & 32 \\
\hline 26 & 10 & 22 & 0.07 & 0.15 & 32 \\
\hline 27 & 21 & 22 & 0.01 & 0.02 & 32 \\
\hline
\end{tabular}


Table 7 continued

\begin{tabular}{llllll}
\hline Line \# & From node \# & To node \# & Resistance (p.u.) & Reactance (p.u.) & $f_{\ell}^{0}$ (MVA) \\
\hline 28 & 15 & 23 & 0.10 & 0.20 & 16 \\
29 & 22 & 24 & 0.12 & 0.18 & 16 \\
30 & 23 & 24 & 0.13 & 0.27 & 16 \\
31 & 24 & 25 & 0.19 & 0.33 & 16 \\
32 & 25 & 26 & 0.25 & 0.38 & 16 \\
33 & 25 & 27 & 0.11 & 0.21 & 16 \\
34 & 28 & 27 & 0.00 & 0.40 & 65 \\
35 & 27 & 29 & 0.22 & 0.42 & 16 \\
36 & 27 & 30 & 0.32 & 0.60 & 16 \\
37 & 29 & 30 & 0.24 & 0.45 & 16 \\
38 & 8 & 28 & 0.06 & 0.20 & 32 \\
39 & 6 & 28 & 0.02 & 0.06 & \\
\hline
\end{tabular}

\section{References}

Alvarado, F., \& Oren, S. (2002). Transmission system operation and interconnection. In National transmission grid study - Issue papers (pp. A1-A35), U.S. Department of Energy.

Borenstein, S., Bushnell, J., \& Stoft, S. (2000). The competitive effects of transmission capacity in a deregulated electricity industry. RAND Journal of Economics, 31(2), 294-325.

Bushnell, J., \& Stoft, S. (1996). Electric grid investment under a contract network regime. Journal of Regulatory Economics, 10(1), 61-79.

California ISO. (2004). Transmission Economic Assessment Methodology (TEAM). Available at www.caiso.com/docs/2003/03/18/2003031815303519270.html

Cardell, J., Hitt, C., \& Hogan, W. (1997). Market power and strategic interaction in electricity networks. Resource and Energy Economics, 19, 109-137.

Craft, A. (1999). Market structure and capacity expansion in an unbundled electric power industry. Ph.D. dissertation, Department of Engineering-Economic Systems and Operation Research, Stanford University, Stanford, USA.

Hobbs, B. (2001). Linear complementarity models of Nash-Cournot competition in bilateral and POOLCO power markets. IEEE Transactions on Power Systems, 16(2), 194-202.

Joskow, P., \& Tirole, J. (2000). Transmission rights and market power on electric power networks. RAND Journal of Economics, 31(3), 450-487.

Latorre, G., Cruz, R., Areiza, J., \& Villegas, A. (2003). Classification of publications and models on transmission expansion planning. IEEE Transactions on Power Systems, 18(2), 938-946.

Murphy, F., \& Smeers, Y. (2005). Generation capacity expansion in imperfectly competitive restructured electricity markets. Operations Research, 53(4), 646-661.

Oren, S. (1997). Economic inefficiency of passive transmission rights in congested electricity systems with competitive generation. The Energy Journal, 18, 63-83.

Oren, S., Gross, G., \& Alvarado, F. (2002). Alternative business models for transmission investment and operation. In National transmission grid study-Issue papers (pp. C1-C36). U.S. Department of Energy.

Sheffrin, A. (2005). Gains from trade and benefits of transmission expansion for the IEEE Power Engineering Society. Proceedings of the IEEE Power Engineering Society 2005 general meeting. San Francisco, USA: Track 5.

Stoft, S. (1999). Financial transmission rights meet Cournot: How TCCs Curb market power. The Energy Journal, 20(1), 1-23.

Yao, J., Oren, S., \& Adler, I. (2004). Computing Cournot equilibria in two settlement electricity markets with transmission constraints. Proceedings of the 37th Hawaii international conference on systems sciences (HICSS). Big Island, Hawaii, USA. 20051b. 